\title{
Severe coronary artery ectasia in a transgender woman undergoing cross-sex hormonal therapy for gender affirmation
}

\begin{abstract}
Cross-Sex Hormone Therapy (CSHT)is an increasing trend among transgender individuals that positively aids both physically and psychologically during gender affirmation. However, there is a lack of data of prolonged CSHT on cardiac health. We report a CSHT-treated transgender woman who presented with significant diffuse coronary artery aneurysmal disease (or coronary artery ectasia).
\end{abstract}

Keywords: coronary artery aneurysm, aneurysm, transgender, transgender woman, cross-sex hormonal therapy, gender affirmation
Volume 10 Issue 5 - 2020

\section{Farhan Chaudhry,' TejeshwarBawa,' Elana Folbe,' Ellen E Kjoller,' Phillip D Levy,' Jahan Q Zeb ${ }^{2}$}

'Department of Emergency Medicine and Integrative Biosciences Center, Wayne State University, USA

${ }^{2}$ Mercy Clinic Cardiology, Mercy Hospital-Joplin, USA

\begin{abstract}
Correspondence: Farhan Chaudhry, Department of Emergency Medicine and Integrative Biosciences Center,Wayne State University, USA, Tel + |-3 | 3-745-3330, Fax 3|3-993-7703, Email gf963@wayne.edu
\end{abstract}

Received: September 20, 2020 | Published: September 28, 2020
Abbreviations: ED, emergency department; STEMI, STelevation myocardial infarction; CSHT, cross-sex hormonal therapy; CVD, cardiovascular disease; CAE, coronary artery ectasia; LCX, left circumflex; LAD, left anterior descending; LM, left main; RCA, right coronary artery; LCA, left coronary artery; DAPT, dual-antiplatelet therapy ; MI, myocardial infarction

\section{Introduction}

The number of patients seeking cross-sex hormonal therapy (CSHT) for gender reaffirmation continues to grow rapidly across the globe. 1 Even though cardiovascular disease is the leading cause of death among transgender individuals after suicide, there have been few published reports or studies relating to the impact of prolonged CSHT on heart health. ${ }^{1}$ Transgender women, in particular, suffer from worse cardiovascular outcomes compared to cisgender woman after adjusting for comorbidities. ${ }^{2}$ There is a significant clinical need to better understand the effects of CSHT in this vulnerable population. We encountered a severe case of diffuse coronary artery aneurysmal disease (or coronary artery ectasia, CAE) that developed in a transgender woman (male-to-female) who was on CSHT for more than 20years.

\section{Case presentation}

A 51-year-oldtransgenderwoman with a history of atrial fibrillation, diabetes mellitus type II, cerebrovascular disease, dyslipidemia, hypertension and a 30 pack-year smoking history presented to the emergency department (ED) with retrosternal chest discomfort that started approximately 12 hours prior to arrival and progressively worsened throughout the day. The patient was hemodynamically stable and did not appear in significant distress. However, a subsequent electrocardiogram (ECG) showed an inferolateral wall ST-elevation myocardial infarction (STEMI) resulting in immediate cardiac catheterization. A troponin obtained on arrival was elevated at $2.41 \mathrm{ng} / \mathrm{mL}$.

\section{Medical history}

The patient had been on CSHT for over 20 years and suffered from multiple CVD risk factors. At-home-medications included diltiazem $10 \mathrm{mg}$, lisinopril 40mg, insulin glargine, and lovastatin 20mg. Patient used spironolactone $100 \mathrm{mg}$ twice daily, as well as estradiol $0.1 \mathrm{mg} / 24$ patch with two patches applied every week and estradiol tablets $0.5 \mathrm{mg}$ for CSHT.

\section{Procedural findings}

Cardiac catherization showed:1) diffuse coronary artery ectasia (CAE) with a chronic occlusion at the mid- left circumflex (LCX), 2) large caliber left main (LM) with mild luminal abnormalities, 3) large caliber left anterior descending (LAD) with diffuse aneurysms proximally and a large aneurysmal diagonal branch, 4) an aneurysmal right coronary artery (RCA) with an occlusion at the mid segment near the bifurcation of the small marginal branch and 5) a severely diffuse aneurysmal right common femoral artery with moderately diffuse plaques. (Figure 1)
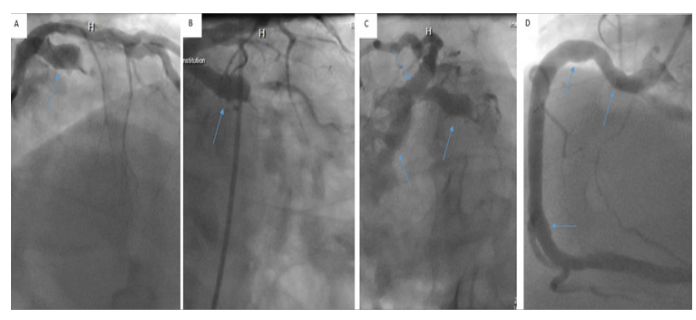

Figure I Blue arrows indicate aneurysms. (A) Left coronary artery (LCA) cranial right anterior oblique view. (B) LCA right anterior oblique view. (C) LCA Spider-View and (D) RCA left anterior oblique view. 


\section{Differential diagnosis}

Severe CAE from severe systemic atherosclerosis formation.

\section{Intervention}

For LCX occlusion, a 4.0x12mm Apex Emerge Balloon was positioned across the occlusion and inflated at nominal atmospheres, and then repositioned to be inflated again at 3 atmospheres. Subsequent angiography did not show improvement of flow. A similar attempt was performed on the occlusion at the mid segment of the small marginal branch of the RCA without any success. Due to risk of perforation of these diffusely large aneurysms in the coronary arteries, further interventions were not performed.

\section{Management}

The patient presents with severe CAE of multiple coronary vessels leading to STEMI. Multiple coronary interventions were attempted but failed and the patient was therefore medically managed with dual antiplatelet therapy (DAPT) and a high intensity statin. An ECG obtained prior to discharge did not show any signs of STEMI and the patient was hemodynamically stable. Echocardiography performed prior to discharge showed lateral wall akinesis of the left ventricle and a mid-range ejection fraction of $40-45 \%$ suggestive of heart failure with moderately reduced ejection fraction. Lisinopril 40mg was maintained to prevent adverse cardiac remodeling associated with heart failure.

\section{Follow-up}

At discharge, patient was advised to stop CSHT pending CVD risk reduction. She was instructed, in addition to continuing current home medications, to replace her lovastatin with atorvastatin $80 \mathrm{mg}$, and to start taking clopidogrel $75 \mathrm{mg}$, nitroglycerin $0.4 \mathrm{mg}$, and rivaroxaban $15 \mathrm{mg}$. One-year later patient remained asymptomatic after treatment and has not required any follow-up echocardiograms, angiograms or stress tests. Patient remains off CSHT and has decided not to restart her CSHT regimen.

\section{Discussion}

CSHT for transgender women often incorporate antiandrogen therapy combined with lifelong estrogen supplementation. CSHT is associated with improved mental health in transgender individuals; younger transgender individuals who present earlier in life often have fewer mental health concerns.1As CSHT usage increases and as more patients become comfortable identifying as transgender, clinicians should be aware that both transgender women and transgender men suffer from significantly greater CVD risk as compared to that seen in the cisgender population. ${ }^{2,3} \mathrm{~A}$ cross-sectional study of surveys showed that transgender women suffer from significantly greater rates of myocardial infarctions(MIs) compared to cisgender woman, but not compared to cisgender men after controlling for comorbidities such as smoking and diabetes. ${ }^{2}$ However, this analysis failed to incorporate information regarding CSHT prevalence, and thus the implication of CSHT on cardiovascular health remains unknown.

One study identified significantly greater risks of cardiovascularrelated mortality in transgender individuals as compared to birth-sex matched controls after gender affirmation. ${ }^{4}$ It is not clear as to whether this is due to CSHT or due to an increased prevalence of known CVD risk factors such as diabetes and smoking within the transgender population. It is known that different forms of estrogen therapy have been associated with variable effects on CVD risk factors and outcomes. For example, oral ethinyl estradiol increases the risk for CVD by augmenting CVD risk factors and thereby increases mortality risk. ${ }^{5,6}$ However, transdermal estrogen therapy has not been shown to be as strongly associated with CVD, and haseven been shown to reduce the risk for venous thromboembolism formation as compared to oral estrogen therapy. ${ }^{5,7}$ Therefore, clinicians should consider transdermal estrogenover oral ethinyl estradiol to reduce CVD risk for CSHT, especially fortrans gender patients who have multiple CVD risk factors.

Currently, the ongoing Gender Dysphoria Treatment in Sweden (GETS) study seeks to clarify the effects of CSHT on vascular structure. ${ }^{8}$ However, as of now, the consequential effects of prolong CSHT on vascular structure remain controversial. This case study reports a potential consequence of prolonged CSHT in a transgender woman that could have resulted in the significant CAE.

Coronary aneurysmsare focal dilations of the coronary vascular lumen greater than 1.5 times compared to adjacent normal segments. ${ }^{9}$ The presence of diffuse coronary artery aneurysms has recently been reclassified as CAE even though, historically, coronary artery aneurysm and ectasia have been used interchangeably. ${ }^{10,11}$ Regardless, both forms are often incidentally found by imaging and are often asymptomatic. When symptomatic, i.e. presenting with angina, coronary aneurysms can be associated with rupture and sudden death. Overall incidence of focal coronary artery aneurysms ranges from $0.3 \%$ to $5.3 \%$ with the incidence of giant coronary aneurysms (diameter greater than $4 \mathrm{~cm}$ ) as low as $0.02 \%$. There was a previous report of a rare non-atherosclerotic RCA giant aneurysm in an older transgender woman resulting in cardiothoracic surgery. ${ }^{12}$ Another case report described a rare finding of both fibromuscular dysplasia and superior mesenteric artery aneurysms in a transgender woman with a history of gender affirmation surgery and CSHT for more than 10 years. ${ }^{13}$ Recent reports of rare aneurysmal formations in transgender women necessitate further study of a potential link between CSHT and aneurysm formation.

CAE is relatively more common, accounting for $5 \%$ of coronary angiograms, and is often secondary to significant atherosclerosis. ${ }^{9}$ This case report presents a transgender woman who suffered a severe case of multiple vessel CAE and aneurysmal disease of the femoral arteries. Her CAE is most likely secondary to atherosclerosis, smoking, and diabetes mellitus; however, her CAE may have been augmented by prolonged CSHT usage.

Sex hormones have a varying effect on vasculature in men and women. ${ }^{14}$ Natural estrogen in premenopausal women has been shown to attenuate aneurysms, while estrogen deficiency in postmenopausal women promotes aneurysms and rupture. ${ }^{15,16}$ Therefore, it is widely believed that estrogen has protective effects on vasculature. However, it should be noted that there are predominately two estrogen receptor (ER) subtypes present in human arteries (ER $\alpha$ and ER $\beta$ ), and the implication of these receptors in atherogenesis is variable.14For example, one rat model indicated that ERawas the sole receptor needed for protection from abdominal aneurysms in the presence of estrogen. ${ }^{17}$ Another mouse model showed that both ER $\alpha$ and ER $\beta$ have atheroprotective potential by preventing calcification in advanced atherosclerotic lesions. ${ }^{18}$ Yet, it was shown that the presence of excess estrogen in ER-suppressed mice promoted atherogenesis. ${ }^{18}$ Thus, it has been hypothesized that estrogen primarily promotes atheroprotection only in the presence of a sufficient number of ERs. 
If there is a deficiency of ERs compared to available estrogen molecules, the excess estrogen molecules may bind to other estrogenbinding proteins thereby increasing transcription of pro-atherogenic molecules. ${ }^{19}$ It has been found that women express $80 \%$ more ER $\alpha$ receptors in arteries than that seen in men, so it can be further hypothesized that excess estrogen in a biologic male (who has less available ERs) could actually promote atherogenesis. ${ }^{17}$

Androgen also plays a complex role in vascular function.14 Some studies have identified that androgen deficiency in human males results in endothelial dysfunction and accelerated rates of atherosclerosis. ${ }^{20,21}$ Yet, activation of androgen receptors in abdominal aortas have been shown to promote endothelial dysregulation leading to aneurysms. ${ }^{22}$ Interestingly, physiologic hyperandrogenemia in and of itself in men and women is not associated with an increased risk of CVD. ${ }^{23-25}$ Therefore, we can hypothesize that maintaining a physiological balance of sex hormones is potentially crucial to reduce vascular damage and thus prevent adverse cardiovascular events.

It should be of note, that our patient has an extensive personal history of serious risk factors that are commonly associated with significant atherosclerosis formation leading to CAE, including long-term smoking and diabetes mellitus. Atherosclerosis is a wellestablished cause of diffuse CAE and it is thus difficult to determine if CSHT may have possibly contributed to atherogenesis in this patient. Nonetheless, transgender patients are at a significantly increased risk for CVD compared to cisgender patients. Our findings, along with a handful of other studies, suggest that prolonged hormone imbalance stemming from CSHT may be a factor promoting atherogenesis.

In general, transgender women have a higher prevalence of CAE risk factors, such as hypertension, hyperlipidemia, diabetes mellitus, and smoking. Therefore, transgender patients are at a significantly greater risk for CVD as compared to that seen in the cisgender population. We believe that transgender individuals should be routinely screened for CVD risk factors such as diabetes mellitus, hyperlipidemia, and smoking. ${ }^{1,3}$ Efforts to aggressively manage modifiable risk-factors to reduce the incidence of CVD should be initiated immediately in this especially vulnerable population. While further studies are needed to better understand the impact of CSHT on cardiovascular health, we recommend a baseline assessment of CVD risk factors for all transgender individuals prior to initiation of CSHT.

\section{Funding support}

Farhan Chaudhry's research fellowship was partially funded by the Society of Academic Emergency Medicine Foundation and the Jewish Fund.

\section{Acknowledgments}

None.

\section{Conflicts of interest}

The authors have no conflicts of interest to declare.

\section{References}

1. Nobili A, Glazebrook C, Arcelus J. Quality of life of treatment-seeking transgender adults: A systematic review and meta-analysis. Rev Endocr Metab disord. 2018;19(3):199-220.

2. Alzahrani T, Nguyen T, Ryan A, et al. Cardiovascular Disease Risk Factors and Myocardial Infarction in the Transgender Population. Circ Cardiovasc Qual Outcomes. 2019;12(4):e005597.
3. Dutra E, Lee J, Torbati T, et al. Cardiovascular implications of genderaffirming hormone treatment in the transgender population. Maturitas. 2019; $129: 45-49$.

4. Dhejne $\mathrm{C}$, Lichtenstein $\mathrm{P}$, Boman $\mathrm{M}$, et al. Long-Term Follow-Up of Transsexual Persons Undergoing Sex Reassignment Surgery: Cohort Study in Sweden. PLoS ONE. 2011;6(2):e16885.

5. Gooren LJ, Wierckx K, Giltay EJ. Cardiovascular disease in transsexual persons treated with cross-sex hormones: reversal of the traditional sex difference in cardiovascular disease pattern. Eur J Endocrinol. 2014;170(6):809-819.

6. Asscheman H, Giltay EJ, Megens JAJ, et al. A long-term follow-up study of mortality in transsexuals receiving treatment with cross-sex hormones. Eur J of Endocrinology. 2011;164(4):635-642.

7. Mohammed K, Abu Dabrh AM, Benkhadra K, et al. Oral vs Transdermal Estrogen Therapy and Vascular Events: A Systematic Review and MetaAnalysis. J Clin Endocrinol Metab. 2015;100(11):4012-4020.

8. Wiik A, Andersson DP, Brismar TB, et al. Metabolic and functional changes in transgender individuals following cross-sex hormone treatment: Design and methods of the GEnder Dysphoria Treatment in Sweden (GETS) study. Contemporary Clinical Trials Communications. 2018;10:148-153.

9. Abou Sherif S, Ozden Tok O, Taskoylu O, et al. Coronary Artery Aneurysms: A Review of the Epidemiology, Pathophysiology, Diagnosis, and Treatment. Front Cardiovasc Med. 2017;4:24.

10. Luo Y, Tang J, Liu X, et al. Coronary Artery Aneurysm Differs From Coronary Artery Ectasia: Angiographic Characteristics and Cardiovascular Risk Factor Analysis in Patients Referred for Coronary Angiography. Angiology. 2017;68(9):823-830.

11. Kawsara A, Nunez Gil IJ, Alqahtani F, et al. Management of Coronary Artery Aneurysms. JACC Cardiovasc Interv. 2018;11(13):1211-1223.

12. Ramirez JL, Kratz JR, Wieselthaler GM. Giant right coronary artery aneurysm presenting as cardiac tamponade. Interact Cardiovasc Thorac Surg. 2018;27(5):787-789.

13. Shalan A, Hughes M, Nicholls M, Thompson A. A Challenging Case of Fibromuscular Dysplasia in a Transgender Patient: Is There a Hormonal Link? EJVES Short Rep. 2018;39:16-19.

14. Boese AC, Kim SC, Yin K-J, et al. Sex differences in vascular physiology and pathophysiology: estrogen and androgen signaling in health and disease. Am J of Physiol Heart and Circ Physiol. 2017;313(3):H524-H545.

15. De Rooij NK, Linn FHH, Van Der Plas JA, et al. Incidence of subarachnoid haemorrhage: a systematic review with emphasis on region, age, gender and time trends. J Neurol Neurosurg Psychiatry. 2007;78(12):1365-1372.

16. Hoh BL, Rojas K, Lin L, et al. Estrogen Deficiency Promotes Cerebral Aneurysm Rupture by Upregulation of Th17 Cells and Interleukin-17A Which Downregulates E-Cadherin. J Am Heart Assoc. 2018;7(8).

17. Laser A, Ghosh A, Roelofs K, et al. Increased estrogen receptor alpha in experimental aortic aneurysms in females compared with males. J Surg Res. 2014;186(1):467-474.

18. McRobb LS, McGrath KCY, Tsatralis T, et al. Estrogen Receptor Control of Atherosclerotic Calcification and Smooth Muscle Cell Osteogenic Differentiation. Arterioscler Thromb Vasc Biol. 2017;37(6):1127-1137.

19. Nofer JR. Estrogens and atherosclerosis: insights from animal models and cell systems. J Mol Endocrinol. 2012;48(2):R13-29.

20. Cai JJ, Wen J, Jiang WH, et al. Androgen actions on endothelium functions and cardiovascular diseases. J Geriatr Cardiol. 2016;13(2):183-196.

21. English K. Men with coronary artery disease have lower levels of androgens than men with normal coronary angiograms. European Heart Journal. 2000;21(11):890-894. 
22. Huang C-K, Luo J, Lai K-P, et al. Androgen Receptor Promotes Abdomina Aortic Aneurysm Development via Modulating Inflammatory Interleukin$1 \alpha$ and Transforming Growth Factor- $\beta 1$ Expression. Hypertension. 2015;66(4):881-891.

23. Calderon-Margalit R, Schwartz SM, Wellons MF, et al. Prospective Association of Serum Androgens and Sex Hormone-Binding Globulin with Subclinical Cardiovascular Disease in Young Adult Women: The "Coronary Artery Risk Development in Young Adults" Women's Study. 2010;95(9):4424-4431.
24. Meun C, Franco OH, Dhana K, et al. High androgens in postmenopausal women and the risk for atherosclerosis and cardiovascular disease: the Rotterdam Study. J Clin Endocrinol Metab. 2018;103(4):1622-1630.

25. Muller M. Endogenous Sex Hormones and Progression of Carotid Atherosclerosis in Elderly Men. Circulation. 2004;109(17):2074-2079. 